Gynäk. Rdsch. 1987;27(suppl. 2):I-XII

\title{
Contents, Vol. 27, Supplement 2, 1987
}

\section{Inhalt}

Editorial XI

1. Hauptthema: Gynäkologische Strahlentherapie - Uterusmalignome

Hauptreferat

Rotte, K.: Die Bedeutung der Strahlentherapie fur die Behandlung des Kollum-

karzinoms 1

Koreferate

Kucera, H.: Die Bedeutung der Strahlentherapie fur die Behandlung des Korpuskarzinoms 11

Pickel, H.: Die Ausbreitung der Uterusmalignome im Lichte der Therapieplanung . 17

Freie Vorträge: Gynäkologische Strahlentherapie

Drack, G.; Haller, U.; Iklé, F.A.; Seelentag, W.: Individualisierte Brachytherapie des

Zervixkarzinoms auf der Basis der Stockholmer-Methode mit dem Selectron

Nachladegerät

21

Hetzel, H.; Fuith, L.C.: Die Entwicklung der Afterloading-Technik (LDR und HDR)

zur Behandlung des Zervixkarzinoms an der Universitäts-Frauenklinik Inns

bruck 22

Hetzel, H.; Url, M.: Dosisspezifikation bei der Brachy-Hochvolttherapie des Zervix

karzinoms (Vergleich Radium-LDR - HDR-Afterloading-Therapie) 26

Vahrson, H.; Rauthe, G.; Römer, G.: Ergebnisse der HDR-Afterloading-Bestrahlung im Vergleich zur konventionellen Radiumtherapie des Kollumkarzinoms ... 31

Eiter, H.; Schurz, A.R.; Rhomberg, W.: Primäre Strahlentherapie des Kollumkarzi noms: 6-jährige Erfahrung mit der Caesium-Afterloading-Therapie bei einer mittleren Dosisrate 34

Behrendt, W.; Bremser, H.; Weber, R.: KJinische Beobachtungen bei der kombinierten Iridium-Afterloading/Perkutan-Bestrahlung beim Korpuskarzinom .... 36

Inhalt

IV

Rauthe, G.; Vahrson, H.; Römer, G.: Vergleich der HDR-Afterloading-Bestrahlung mit der Radium-Packmethode beim Korpuskarzinom 39

Voss, A.-C; Warnecke, H.-H.: Anpassung der Perkutanbestrahlung an die Afterloading-Therapie beim nicht operierten Kopus- und Zervixkarzinom

Gerstner, G.J.; Kucera, H.; Kudlacek, St.; Micksche, M.: Zelluläre Immunreaktionen bei Patientinnen mit Korpuskarzinom - Einfluß der Strahlentherapie

Enzelsberger, H.; Metka, M.; Salzer, H.: Der Einfluß von parenteraler Ozon-Sauerstoíf-Gemisch-Applikation auf den Immunstatus bei Patientinnen mit Zervix karzinom $\quad 45$

Fuith, L.C.; Daxenbichler, G.; Hetzel, H.; Dapunt, O.: Effekte der Strahlentherapie auf den Serumspiegel des Tumorantigens SCC bei Kollumkarzinomen 
Meier, W.; Eiermann, W.; Stieber, P.; Fateh-Moghadam, A.; Hepp, H.: Erfahrungen mit dem Tumormarker SCC, insbesondere zur Therapieerfolgskontrolle beim Zervixkarzinom (Op und Radiatio) 50

Kölbl, H.; Kirchheimer, J.C.; Christ, G.; Tatra, G.: Plasminogenaktivatoren bei Uterusmalignomen $\quad 52$

Steck, T.; Gille, J.; Caffier, H.; Wacker, S.: Immunzytochemischer Nachweis des

Östrogenrezeptors im Endometriumkarzinom 54

Reitzenstein, M.; Kirschner, M.; Tulusan, A.H.: Rezidivhäufigkeit und Überlebenszeit beim Endometriumkarzinom in Abhängigkeit von histologischen Krite-

rien 55

Herzog, R.E.; Lang, S.; Seufert, R.: Das DNS-Histogramm als Prognosefaktor des Endometriumkarzinoms 57

2. Hauptthema: Gynäkologische Lasertherapie

Hauptreferat

Janisch, H.; Dadak, Ch.: Chirurgische C $\theta 2$-Lasertherapie in der Gynäkologie

(Physik, biologische Wirkung, Sicherheitsvorkehrungen) 59

Koreferat

Albrich, W.: Klinische Anwendung von Lasersystemen in der Gynäkologie $\quad 66$ Mitteilungen zum 2. Hauptthema

Hosmann, J.; Laufer, G.; Czerwenka, K.; Wollenek, G.; Reinold, E.: Biointeraktionen zwischen Excimer Laser Energie und Tubengewebe - mögliche Hinweise für praktische Anwendbarkeit 73

Keckstein, J.; Wolf, A.; Kirchmayr, R.: Die Anwendung des ND-YAG-Lasers im Rahmen der operativen Pelviskopie 74

Wolf, A.S.; Keckstein, J.; Kirchmayr, R.: Therapiekonzept der Endometriose unter Einschluß der Laser-Chirurgie $\quad 76$

Schieder, K; Dadak, Ch.; Kölbl, H.; Janisch, H.: Die Früh-Second-Look Laparoskopie nach sterilitätschirurgischen Eingriffen mit dem C02-Laser $\quad 78$

Inhalt IX

Untch, M.; Eiermann, W.; Harbeck, N.; Bartl, R.; Hepp, H.: Immunzytochemischer Nachweis von Tumorzellen im Knochenmark bei Mammakarzinompatientinnen (Primärtherapie) 296

Diergarten, K.; Eiermann, W.; Scheidel, P.; Schüßler, B.: Rekonstruktion der Brust nach modifiziert radikaler Mastektomie mit der Haut-Expandertechnik .... 298

Merkle, E.: Adjuvante Chemotherapie des Mammakarzinoms $\quad 300$

Christmann, D.: Mitoxantron als Monotherapie beim metastasierten Mammakarzinom 303

Freie Vorträge: Poster

Heytmanek, G.; Enzelsberger, H.; Schurz, B.; Metka, M.: Management des klimakterischen Syndroms 305

Heytmanek, G.; Metka, M.; Enzelsberger, H.; Schurz, B.; Kovarik, J.: Beziehung verschiedener Knochenumsatzparameter zu den Ergebnissen der Knochendichtemessung 306

Enzelsberger, H.; Metka, M.; Heytmanek, G.; Schurz, B.: Der Einfluß oraler KontrazeptivaaufdieKnochendichtebeiklimakterischenFrauen $\quad 309$

Metka, M.; Heytmanek, G.; Schurz, B.; Enzelsberger, H.; Spona, J.: Beziehung ver- 
schiedener Hormonparameter zu den Ergebnissen der Knochendichtemessung 312 Metka, M.; Heytmanek, G.; Enzelsberger, H.; Kurz, Ch.; Baumann, B.: Der Gelenksschmerz in der Prä- und Postmenopause 314

Egarter, Ch.; Schurz, B.; Manavi, M.; Grünberger, W.: Endokrinologische Störungen beiMayer-Rokitansky-Küster-Syndrom 316

Lang, U.; Römer, G.; Vahrson, H.: Rationelle Rezidiv- und Metastasendiagnostik im kleinen Becken durch Stanzbiopsie mit der Tru-Cut@-Punktionskanüle . . 317 Gerstner, G.J.; Kronich, W.; Müller, G.: Antibiotika-Prophylaxe mit Ciprofloxacin bei Hysterektomien - Erste Ergebnisse 319

Seufert, R.; Casper, F.; Herzog, R.; Heidler, H.: Die Rolle der quergestreiften Sphinctermuskulatur fur den Hamröhrenverschluß unter Belastung 322 Lentsch, P.; Dierkopf, W.; Hesse, U.; Schüßler, B.: Die Therapie der komplizierten Harnstauungsniere in der Schwangerschaft 323

Heidegger, H.; Strobel, G.; Schulz, W.; Rebstöck, W.: Die SDS-PAA Elektrophorese der Urinproteine; eine Methode in der geburtshilflich-nephrologischen Überwachung der schwangerschaftsinduzierten Hypertonie 326

Bülau, A.;Niebauer, B.: Inzidenz der Chlamydieninfektionen bei Graviden 329

Gerstner, G.J.; Müller, G.; Nahler, G: Die Behandlung der asymptomatischen Bakteriurie in der Gravidität mit Amoxicillin als Einmal- oder 4-Tages-Therapie eine randomisierte Studie 331

Loos, W.; von Hugo, R.: Klinische und pathobiochemische Befunde beim HELLPSyndrom $\quad 333$

Wiesner, F.; Jäger, W.; Bauer, U.: Das HELLP-Syndrom - Diagnostik und Therapie 334 Voigt, H.J.; Singer, H.; Faschingbauer, Ch.: Intrauterine Therapie bei fetaler supraventrikulärer Tachykardie und Hydrops fetalis 336

Tabarelli, M.; Ka甲ellus, E.; Huter, O.; Krause, P.; Ebner, A.; Madersbacher, H.: Ergebnisse einer Sterilitätstherapie durch homologe Insemination bei Paaren mit Paraplegie des Gatten 339

Inhalt

$\mathrm{V}$

Walz, K.-A.; Fischer, P.: Der Laser bei gynäkologisch-operativen Kleineingriffen . . 80 Dadak, Ch.; Schieder, K.; Janisch, H.: C $\theta 2$-Lasertherapiebei der Behandlung benigner und maligner Veränderungen der Vulva 82 Krause, P.; Fuith, L.C.; Wartusch, B.; Dapunt, O.: C 02 -Lasertherapie genitaler Kondylome unter Berücksichtigung der Virustypisierung 85

Breuel, C; Adam, R.; Siebzehnrübl, E.; Tulusan, A.H.: Gynäkologische Lasertherapie bei der Behandlung von Condyloma acuminata und Carcinoma in situ im Genitalbereich 88

Bistoletti, P.; Hjerpe, A.; Moreno-Lopez, J.: Genitale Human-Papilloma-VirusInfektion nach Laserkonisation $\quad 90$

3. Hauptthema: Das Plazenta-Insuffizienzsyndrom Hauptreferat

Winter, R.: Ultraschalldiagnostik der fetalen Wachstumsretardierung 93 Koreferate

Kastendieck, E.: CTG-Überwachung bei chronischer Plazentainsuffizienz 99 Lang, N.: Die klinische Bedeutung biochemischer Parameter fur Diagnose und The- 
rapie der chronischen Plazentainsuffizienz 108

Philipp, K.; Skodler, W.D.: Messung der uteroplazentaren Durchblutung mittels

Radioisotopen und Doppler-Ultraschall 116

Hauptreferat

Schröck, R.: Therapie des Plazenta-Insuffizienzsyndroms 121

Sonderreferat Welsch, H.; Krone, H.A.: Sektio-Mortalität und Letalität in Bayern 1983-1986 ...

127

Mitteilungen zum 3. Hauptthema

Voigt, H.J.; Landendörfer, W.; Segerer, H.: Geburtshilfe bei schwerer fetaler

Wachstumsretardierung. Pränatale Diagnostik und geburtshilfliche Resultate .... 133

Niedner, W.; Winkler, U.; Seitzer, D.: Zur kardiotokographischen Plazentainsuffi-

zienzdiagnostik 136

Fitz, R.; Simbruner, G.; Rudelstorfer, R.; Salzer, H.: Der Wärmefluß vom Kopf des

Feten korreliert mit dem $\mathrm{pH}$ des Nabelschnurblutes 138

Rudelstorfer, R.; Feiks, A.; Neunteufel, W.; Bieglmayer, Ch.: Laminin und PlazentaInsuffizienzsyndrom 140

Deichert, U.; Rüschoff, J.; Lammel, R.; Knecht, J.; Baltzer, G.; Sturm, G.: Vergleich der Magnesiumkonzentrationen im Plazentargewebe mit klinischen und endo-

krinologischen Daten 142

Kaufmann, P.; Schweikhart, G.; Mikolajczak, J.: Mophologie der normalen und pathologischen Zottenreifung 144

Inhalt

VI

Mikolajczak, J.; Ruhrberg, A.; Fetzer, M.; Kaufmann, P.; Goecke, C: Irreguläre Zottenreifung bei Frühgeburtlichkeit und Übertragung, und ihre Darstellbarkeit

im Ultraschall 145

Schweikhart, G.; Kaufmann, P.: Endzottenmangel und klinische Relevanz 147

Beck, Th.; Rosenthal, H.; Weikel, W.: Plazentainsuffizienz bei Gestose: Morphometrische Untersuchungen zur Reifung und Differenzierung der Resorptions-

zotten 148

Marhold, W.; Medl, M.; Leodolter, S.: Zum Problem der Reproduzierbarkeit von

Doppler-Sonographischen-Flow-Messungen während der Schwangerschaft . 151

Skodler, W.D.; Philipp, K.; Pateisky, N.; Sagl, R.; Reinold, E.: Puls-Doppler-Fluß-

messungen bei Zwillingsschwangerschaften 154

Ertl, U.; Hecher, K.; Spernol, R.; Szalay, St.; Haselbach, H.: Duplex-Doppler-Flow-

messungen im 3. Trimenon - eine neue Methode zur Erfassung der Plazenta

insuffizienz 155

Hecher, K.; Ertl, U.; Spernol, R.; Haselbach, H.; Szalay, St.: Klinische Relevanz von

Doppler-Flowmessungen während der Schwangerschaft 161

Kubista, E.; Skodler, W.; Heytmanek, G.: Verbesserung der Plazentaperfusion bei

Doppler-Ultraschall-Messungen nach transkutaner Nervenstimulation .... 164

Kaesemann, H.; Goerlitz, W.: Computergestützte Auswertung von Dopplersequen-

zen in fetalen Gefäßen bei chronischer Plazentainsuffizienz 167

Wisser, J.; Knitza, R.; Krone, S.; Versmold, H.: Erste Erfahrungen mit gepulsten

Doppleruntersuchungen bei Feten mit intrauteriner Mangelentwicklung .... 170

Genger, H.; Enzelsberger, H.; Salzer, H.: Carnitin als Therapie bei ambulanten 
Patientinnen mit Plazentainsuffizienz

173

Feiks, A.; Nowotny, C; Rudelstorfer, R.; Gruber, W.: Rheographische Befunde unter Therapie der Plazentainsuffizienz 175

Paulick, R.; Kastendieck, E.; Weth, B.; Wernze, H.: Kritische Grenze für einen bedrohlichen Sauerstoffmangel beim Feten gemessen an der sympathoadrenalen Reaktion und der Entwicklung einer Azidose 178

Schuhmann, R.; Halberstadt, E.: Biochemische und biophysikalische Änderungen bei Nabelschnurkompressionen (tierexperimentelle Untersuchungen) 180 Kirschbaum, M.; Bödeker, R.H.; Künzel, W.: Experimentelle intrauterine Asphyxie und ihre Auswirkung auf die Blut-Hirn-Schranke im Großhirn des Meerschweinchens 183

Hohmann, M.; Künzel, W.: Die Wirkung von Dihydroergotamin auf die Uterusdurchblutung trächtiger Meerschweinchen 185

Freie Vorträge: IVF, Pränatale Diagnostik

Reinthaller, A.; Bieglmayer, Ch.; Deutinger, J.; Csaicsich, P.; Riss, P.; Fischl, F.; Müller-Tyl, E.: Progesteron und 17-Hydroxyprogesteron als zusätzliche Para meter zur Zyklusüberwachung für die in-vitro-Fertilisierung 187 Urdl, W.; Winter, R.; Pusch, H.; Hönigl, W.; Mayer, H.O.: Verbesserung von IVFResultaten durch die Anwendung einer Kombination von HMG und FSH zur ovariellen Hyperstimulation 189

Tabarelli, M.; Martin, J.; Haid, Ch.; Karpellus, E.; Marth, Ch.; Zech, J.; Daxenbichler, G.: In-vitro-Fertilisierung: Vaginale Follikelpunktion in Lokalanästhesie . 191 Inhalt VII

Würfel, W.; Kriismann, G.; Rothenaicher, M.; Krüsmann, W.: In-vitro-Fertilisation und intratubarer Embryotransfer - Erste Erfahrungen und derzeitige Stellung derMethode 194

Kessler, H.H.; Dohr, G.; Motter, W.; Desoye, G.; Winter, R.; Urdl, W.; Pusch, H.H.; Ziegler, A.: Immunologische Aspekte des heterologen Embryotransfers ... 197 Terinde, R.; Keckstein, J.; Jonatha, W.D.; Tschiirtz, S.: Erweiterte Pränataldiagnostik durch ultraschallkontrollierte Fetalblutgewinnung 199

Zahn,F.W.: Chorionbiopsie versus Amniozentese 202

Berg, D.: Die Frequenz von genetisch indizierten Fruchtwasseruntersuchungen in Bayern - Daten der BPE $1985 \quad 203$

Freie Vorträge: Geburtshilfe

Rempen, A.; Feige, A.: Vaginale Sonographie der normalen Frühgravidität

Hofmann, H.M.H.; Urdl, W.; Hönigl, W.: Die zervikale Schwangerschaft - Dia gnose und Therapie 206

Hägele, D.; Berg, D.; Lack, N.; RienhofF, O.: Zur Wirksamkeit von Perinatalerhebungen 208

Knitza, R.; Linke, M.; Wisser, J.; Versmold, H.; Hepp, H.: Medikamentöse RDSProphylaxe. Eine Befragung deutscher Frauenkliniken 210

Schwiersch, U.; Voigt, H.-J.; Eydam, P.; Segerer, H.; Tulusan, A.H.: Geburtshilfliches Management und perinatale Mortalität und Morbidität frühgeborener Kinder zwischen 500 und 2000 Gramm 213

Ralph, G.; Lichtenegger, W.; Kopera, D.; Ipsiroglu, O.: Management beim vorzeitigen Blasensprung 216 
Martius, J.; Hillier, S.L.; Krohn, M.J.; Kiviat, N.; Eschenbach, D.A.: Die bakterielle Vaginose und andere Urogenitalinfektionen in der Schwangerschaft: Mögliche Komplikationen 219

Mursch, G.: BEL - geburtshilfliches Management an der Landesfrauenklinik Linz . 220

Schneider, A.; Eiermann, W.; Pfeiffer, U.; Hepp, H.: Geburtsleitung nach vorausgegangener Kaiserschnittentbindung 223

Radivojevic, K; Nasr, F.; Kosian, K.; Rudelstorfer, R.: Alternative Geburt und

Klinikentbindung aus der Sicht werdender Mutter 225

Walcher, W.: Schwangerschaft und Sexualität 227

Placzek, U.: Körpersprache unter der Geburt 230

Kainer, F.; Winter, R.; Hofmann, H.M.H.; Karpf, E.F.: Das Steißteratom - Praepar-

tale Diagnose, Schwangerschaftsverlauf und Geburtsmodus 232

Schramm, Th.; Gloning, K.Ph.; Brusis, E.: Diagnostik und Therapiemöglichkeit bei nicht immunologisch bedingtem Hydrops fetalis 234

Hack, R.; Stöger, H.; Kaiser, W.; Eitelberger, F.: Schwangerschaft und Geburt bei

Niereninsuffizienz 236

von Hugo, R.; GraefГ, H.: Hämorrhagische Komplikation nach gleichzeitiger Anwendung von AT-III-Konzentrat und nieder dosiertem Heparin 239

Lechner, W.; Marth, Ch.: Wehenhemmung mit Theophyllin - experimentelle und klinische Untersuchungen 241

Zahn, V.: ß-Blocker - bei sekundärer Wehenschwäche und als Antidot von ß-Sympathikomimetika 243

Inhalt

VIII

Freíe Vorträge: Gynäkologie

Gerstner, G.J.; Gredler, B.; Friedl, H.P.: Epidemiologie der Uterusmalignome in Österreich 1981-1985 246

Hosmann, J.; Czerwenka, K.; Gitsch, E.: HPV-Subtypisierung mittels SouthernBlot-Technik an Zervixkarzinomen 248

Lahousen, M.; Haas, J.: Adjuvante Chemotherapie beim Zervixkarzinom nach abdomineller Radikaloperation $\quad 250$

Schatten, Ch.; Pateisky, N.; Philipp, K.; Skodler, W.D.: Immunszintigraphie mit monoklonalen Antiköpern - klinische Einsatzmöglichkeiten in der gynäkologischen Onkologie 251

Sevelda, P.; Salzer, H.: Prognostisch bedeutsame Faktoren beim Ovarialkarzinom der Figo-Stadien I und II 253

Schmidl, S.; Sevelda, P.; Genger, H.; Salzer, H.: Erste Erfahrungen und Ergebnisse der intraperitonealen Chemotherapie beim Ovarialkarzinom 254

Schurz, B.; Metka, M.; Eppel, W.; Enzelsberger, H.; Reinold, E.: Vaginosonographie in der Gynäkologie mit speziellem Stellenwert beim Endometriumkarzinom . 256

Sautter, Th.: Die Transvaginalsonographie in der Frauenheilkunde 258

Fronius, G.: Stellenwert der Ultraschalluntersuchung bei der praeoperativen Klärung gynäkologischer Untersuchungsbefunde 260

Kubista, E.; Pawelka, R.: Die Klammernahttechnik als primärer Scheidenverschluß bei abdominaler Operation 262

von Hugo, R.; Potthoff, A.: Befinden und Zufriedenheit nach gynäkologischen Ope- 
rationen 264

Rammer, E.; Friedrich, F.: Die Effektivität der Inkontinenzoperations-Techniken . 266 Kranzfelder, D.; Baumann, A.: Erfolg und Mißerfolg bei der operativen Therapie inkontinenter Frauen. Analyse prä- und postoperativ erhobener morphologischer und funktioneller Befunde 268

Hesse, U.; Dimpfl, T.; Lentsch, P.; Schüßler, B.: Schwangerschaft, Geburt, Streßinkontinenz - eine Kausalkette? 272

Ferstl, F.J.: Röntgenologische Darstellung der anatomischen Veränderungen durch die Harninkontinenz-Operation nach Lahodny 274

Stark, G.: Modifikation der Straßmann'schen Operation 278

Enzelsberger, H.; Eppel, W.; Grünberger, W.: Zur gynäkologischen Entwicklung untergewichtig geborener Mädchen 279

Rost, I.; Bali, Ch.; Irsigler, K.; Leodolter, S.: Das antikonzeptionelle Verhalten von Patientinnen mit Diabetes mellitus 282

Mitterschiffthaler, G.; Theiner, A.; Hetzel, H.; Fuith, L.C.: Propofol-MonoanästhesiezuHDR 284

Schurz, B.; Egarter, Ch.; Kurz, Ch.; Heytmanek, G.; Huber, J.C.; Knogler, W.:

ß-Endorphinbestimmungen im normalen Zyklus bei Zyklusstörungen und im

Klimakterium 287

Wischnik, A.: Neuere Therapieansätze in der Therapie der primären Dysmenorrhoe klinische und in-vitro-Ergebnisse 289

Wachter, I.; Weissenbacher, E.R.; Enders, S.; Ruckdeschl, G.; Braveny, I.; Gutschow, K.: Aktuelle Untersuchungen zum Gardnerella vaginalis Befall 292 de Waal, J.;Baltzer, J.;Ablaßmeier, B.;Kürzl, R.;Messerer, D.; Zander, J.:Histologische Klärung mammographisch suspekter, nicht palpabler Veränderungen der Brust 293 Inhalt

$\mathrm{X}$

Vavra, N.; Fitz, R.; Sevelda, P.; Fuchs, A.R.; Fuchs, F.; Husslein, P.: Lassen Prostaglandin- und Oxytocin-Plasmaspiegel einen Rückschluß auf die zu erwartende Wirksamkeit einer Wehenhemmung bei Frühgeburten zu? 342

Egarter, Ch.; Schurz, B.; Husslein, P.: Erhöhung der Effizienz bei elektiver Geburtseinleitung mit Prostaglandin (PG) E2 345

Mutke, H.G.: Der errechnete Geburtstermin, die Timing-Störung zwischen Mutter und Kind und die Plazentainsuffizienz $\quad 347$

Comely, M.; Reinheimer, G.: Kritische CTG-Befunde zwischen dem Zeitpunkt der Sektio-Entscheidung und dem Operationsbeginn. Vorteil und Probleme der telemetrischen Herztonüberwachung 349

Toussaint, M.-N.; Kaltenbach, F.-J.; Stadthaus, I.: Ergebnisse histomo $\varphi$ hologischer Untersuchungen der Plazentazotten bei EPH-Gestose: Beitrag zur Plazenta insuffizienz 351

Jensen, A.; Lang, U.; Künzel, W.: Die Kopfhautdurchblutung des Feten als SchockIndikator $\quad 361$

Merkle, E.; Tulusan, A.H.; Fuchs, U.: Chemotherapie des metastasierten Mammakarzinoms nach dem FNC-Schema 364

Autorenregister

366 


\section{Editorial}

Es ist uns eine besondere Freude, namens der Österreichischen Gesellschaft für Gynäkologie und Geburtshilfe, unserer bayerischen Schwestergesellschaft die besten Glückwünsche anläßlich ihres 75jährigen Jubiläums zu überbringen. Gleichzeitig feiern wir ein kleines Jubiläum, nämlich die 15. Gemeinsame Tagung unserer beiden Gesellschaften. Die gemeinsamen Jahreskongresse haben sich in der Vergangenheit als außer-ordentlich interessant erwiesen, und die gegenseitige wissenschaftliche Stimulierung war unübersehbar.

Es war der Wunsch und der Vorschlag der Bayerischen Gesellschaft als erstes Hauptthema die gynäkologische Strahlentherapie der Uterus-malignome zu wählen. Die rasante Entwicklung der Radiotherapie, aber ebenso auch der Umstand, daß diese Therapie schon seit 9 Jahren nicht mehr ein Hauptthema unserer gemeinsamen Tagung war, hat die Behand-lung dieser Materie dringend erfordert. Dem Tagungspräsidenten, Herrn Prof. WULF ist es zu danken, die Thematik in 3 Teile zu gliedern, und jeweils einen kompetenten Fachmann einzuladen. Eine sinnvolle Ergän-zung dazu werden die Vorträge zum Hauptthema sein, in denen aus zahlreichen Kliniken und Abteilungen über die Erfahrung berichtet wird.

Das zweite Hauptthema war ein Anliegen beider Gesellschaften, nämlich die gynäkologische Lasertherapie. Mit dieser neuen Technik wurden Möglichkeiten erschlossen, die, wie es sich gezeigt hat, noch lange nicht ausgeschöpft sind. Diese Technik hat aber auch neue Probleme mit sich gebracht, die mit der Physik und biologischen Wirkung in Zusammenhang stehen sowie den daraus resultierenden Sicherheitsvor-kehrungen. Im zweiten Referat werden uns die klinischen Anwendungs-möglichkeiten von verschiedenen Lasersystemen in der Gynäkologie vor-gestellt. Auch hier werden Vorträge zum Hauptthema diesen interessan-ten Bereich in unserem Fachgebiet ergänzen.

Das dritte Hauptthema ist einem vielschichtigen Problem in der Geburtshilfe gewidmet, von dem wir zwar schon viel, aber noch lange nicht alles wissen. Die Bezeichnung

„Plazentainsuffizienzsyndrom”, sie

\section{Editorial}

XII

wurde meines Wissens nach von unserem Tagungspräsidenten, Herrn Prof. WULF, geprägt, spiegelt die Vielfältigkeit dieses Erscheinungsbil-des wieder. Um dieser Tatsache gerecht zu werden, sind insgesamt 5 Referate vorgesehen, die einerseits die Auswirkungen auf den sich entwickelnden Feten (durch die sonographische Biometrie als Parameter des fetalen Wachstums, durch die Kardiotokographie als Parameter des fetalen Zustandes), sowie andererseits biochemische Parameter für Diagnose und Therapie behandeln; ein aktueller Aspekt dieses Syndroms ist die Messung der uteroplazentaren Durchblutung als ein Parameter der Funktion der Plazenta; nicht zuletzt sind die therapeutischen Maßnahmen im Rahmen der Plazentainsuffizienzsyndroms von besonderer Bedeutung.

Die Zahl der angemeldeten Vorträge und Poster hat unsere Erwar-tungen weit überschritten. Eine erfreuliche Tatsache, spricht es doch für den Fleiß und das Interesse unserer jungen Kolleginnen und Kollegen, nicht nur an den Kliniken, sondern auch an den zahlreichen Fachabtei-lungen.

Unser ganz besonderer Dank gilt auch in diesem Jahr den Sponsoren, denn nur mit ihrer Unterstützung war es möglich, das Kongreßsupple-ment auch in diesem Jahr herzustellen. Unser Dank gilt aber auch den Mitarbeitern des Verlages, S. Karger, Basel, sowie der Druckerei Ferdinand Berger, Horn, in NO, für die gute und prompte Zusammenarbeit.
E. Reinold
E. Gitsch 
Für das Zustandekommen dieses Kongreß-Supplementes sind wir folgenden Firmen (in alphabetischer

Reihenfolge) zu großem Dank

verpflichtet:

Fa. BAYER-PHARMA

Fa. GEROT

Fa. CHEMIE-LINZ

Fa. GRÜNENTHAL

Fa. CHEMOMEDICA

Fa. ORGANON

Fa. CILAG

Fa. SCHERING

Ferner danken wir folgenden Firmen (ebenfalls in alphabetischer Reihenfolge) für ihre großzügige Unterstützung:

Fa. BEHRING

Fa. TOSHIBA

Fa. COMESA

sowie den Firmen

Fa. MERKLE

HEWLETT \& PACKARD

Fa. SOLCO

JANSSEN 